\title{
Resiliência e educação laboral: uma abordagem no trabalho da enfermagem do Centro de Material e Esterilização
}

Resilience and occupational education: an approach to nursing at the Material and Sterilization Center

Resiliencia y educación laboral: un enfoque en el trabajo de enfermería en el Centro de Material y Esterilización

\author{
Alcinéa Rodrigues Athanázio \\ ORCID: https://orcid.org/0000-0002-0520-0007 \\ Universidade Federal Fluminense, Brasil \\ E-mail: alcinea_rodrigues@hotmail.com \\ Enéas Rangel Teixeira \\ ORCID: https://orcid.org/0000-0002-1721-2056 \\ Universidade Federal Fluminense, Brasil \\ E-mail: eneaspsi@hotmail.com \\ Benedito Carlos Cordeiro \\ ORCID: https://orcid.org/0000-0001-6387-511X \\ Universidade Federal Fluminense, Brasil \\ E-mail: bcordeiro@id.uff.br \\ Lídia Marina do Carmo Souza \\ ORCID: https://orcid.org/0000- 001-7488-3780 \\ Universidade Federal Fluminense, Brasil \\ E-mail:limarcas@oi.com.br \\ Rose Mary Costa Rosa Andrade Silva \\ ORCID: https://orcid.org/0000-0002-6403-2349 \\ Universidade Federal Fluminense, Brasil \\ E-mail: roserosa@enf.uff.br \\ Eliane Ramos Pereira \\ ORCID: https://orcid.org/0000-0002-6381-3979 \\ Universidade Federal Fluminense, Brasil \\ E-mail: elianeramos.uff@gmail.com
}

\section{Resumo}

Objetivo: apontar a Educação Permanente em Saúde como instrumento de sensibilização para gestores, coordenação de enfermagem e demais profissionais sobre os benefícios institucionais e sociais de um processo educacional ativo. Metodologia: exploratório, abordagem qualitativa, cenário centro de material e esterilização de um hospital universitário. Os participantes, 31 da equipe de enfermagem: 03 enfermeiros, 16 técnicos e 12 auxiliares de enfermagem. Resultados: submetidos à análise de conteúdo desvelando três categorias: Desagregação; Invisibilidade e Enfrentamento. Conclusão: reorientando de forma multidirecional, a percepção sobre as especificidades do trabalhador, apesar da relevância do desempenho profissional, não é vista, devido a laços interpessoais restritos ao trabalho confinado. A Educação Permanente em Saúde, devido ao enfoque inter e multiprofissional, surge como instrumento capaz de produzir transformações nas práticas e contextos de trabalho, visto que a falência do comportamento resiliente pode ser a alienação ou transtornos de ordem biopsicossocial. Requisitam-se outras abordagens.

Palavras-chave: Enfermagem; Esterilização; Educação em saúde; Educação continuada.

\begin{abstract}
Objective: To point out Permanent Health Education as an instrument of awareness for managers, nursing coordination and other professionals about the institutional and social benefits of an active educational process. Methodology: exploratory, qualitative approach, setting the material center and sterilization of a university hospital. The participants, 31 of the nursing staff: 03 nurses, 16 technicians and 12 nursing assistants. Results: subjected to content analysis unveiling three categories: Disaggregation; Invisibility and Coping. Conclusion: reorienting in a multidirectional way, the perception about the specificities of workers, despite the relevance of professional performance, is not seen, due to interpersonal ties restricted to confined work, Permanent Health Education, due to the inter and multiprofessional
\end{abstract}


approach, appears as an instrument capable of producing changes in work practices and contexts, since the failure of resilient behavior can be alienation or biopsychosocial disorders. Other approaches are required.

Keywords: Nursing; Sterilization; Health education; Continuing education.

\begin{abstract}
Resumen
Objetivo: señalar la Educación Permanente en Salud como un instrumento de concienciación para los gerentes, la coordinación de enfermería y otros profesionales sobre los beneficios institucionales y sociales de un proceso educativo activo. Metodologia: enfoque exploratorio, cualitativo, establecimiento del centro de materiales y esterilización de un hospital universitario. Los participantes, 31 del personal de enfermería: 03 enfermeras, 16 técnicos y 12 auxiliares de enfermería. Resultados: sometidos a análisis de contenido revelando tres categorías: Desagregación; Invisibilidad y afrontamiento. Conclusión: reorientando de una manera multidireccional, la percepción sobre las especificidades de los trabajadores, a pesar de la relevancia del desempeño profesional, no se ve, debido a los lazos interpersonales restringidos al trabajo confinado. La educación permanente en salud, debido al enfoque inter y multiprofesional, aparece como un instrumento capaz de producir cambios en las prácticas y contextos de trabajo, ya que el fracaso del comportamiento resiliente puede ser alienación o trastornos biopsicosociales. Son solicitados otros enfoques.
\end{abstract}

Palabras clave: Enfermería; Esterilización; Educación en salud; Educación contínua.

\title{
1. Introdução
}

Resiliência é o termo que exemplifica a adaptação de um organismo às intempéries, alterações ou infortúnios externos (Portela \& Liberalesso, 2015), descrição aceita em disciplinas, como a física e a psicologia (Portela, 2015 \& Cruz et al. 2018), dentre outras. Ela demanda perseverança, sem deixar de reconhecer a fragilidade que é própria à condição do ser vivo (Oviedo \& Czeresnia, 2015). Neste estudo, em que são abordados os estímulos provenientes do trabalho realizado pela equipe de enfermagem, no Centro de Material e Esterilização, de um hospital público, como tema, optou-se pela definição de resiliência, utilizada no contexto psicossocial, referida como a aptidão de um determinado sistema - corpo, que independente da exposição a agentes perturbadores, se permite recuperar (Oviedo \& Czeresnia, 2015; Cecílio et al. 2016; Deuster, \& Silverman, 2013), ressaltando a relevância deste setor.

Nos serviços de saúde, o Centro de Material e Esterilização é o setor responsável pela recepção, limpeza, preparo, esterilização e distribuição de todos os materiais utilizados nos procedimentos invasivos, realizados em pacientes internos ou externos. Este setor possibilita aos profissionais da equipe multidisciplinar de saúde, o suporte necessário à progressão do plano terapêutico, a partir do material esterilizado (Sobecc, 2017; Brasil, 2012; Gil, Camelo \& Laus, 2013).

Entretanto, tal como no contexto mundial, o trabalho no Centro de Material e Esterilização, vem passando por transformações, em decorrência da tecnologia que tem exigido constantes mudanças na sua forma de organização (Fusco \& Spiri, 2014), com atividades distintas em concordância com as normativas legais. Uma vez que no âmbito assistencial, tem-se como real, que muitas intervenções técnicas-curativas bem sucedidas, podem não surtir efeitos positivos, se a recuperação do paciente, for abalada pelo surgimento de uma infecção.

Partindo desses pressupostos, revelam-se como imprescindíveis, os aspectos das atividades desenvolvidas pela equipe de profissionais de enfermagem do Centro de Material e Esterilização, composta por Enfermeiros (as) Técnicos (as) e/ou Auxiliares de Enfermagem, que buscam corresponder às exigências de um trabalho, que trilha pelo caminho da tecnologia a serviço da saúde e da segurança de pacientes e profissionais.

Nesse sentido, são realizadas atividades laborais rotineiras e repetitivas, em espaço confinado e em meio à exposição a riscos físicos, químicos, biológicos e psicossociais, decorrente do anonimato e do afastamento do convívio interpessoal (Silva, Loureiro, Silva, \& Novaes, 2020). É nesse ambiente que a equipe de enfermagem lotada no Centro de Material e Esterilização constrói a segurança no âmbito dos serviços de saúde, cuja necessidade de aporte educacional permanente, se faz presente, para atender às exigências das novas tecnologias em esterilização e processamento de materiais de alto custo financeiro. Assim, justifica-se o estudo relevante frente as condições de trabalho dos profissionais de enfermagem relacionando as possibilidades 
de ampliação do comportamento resiliente frente aos riscos ocupacionais através de estratégias de educação permanente em saúde.

Os profissionais da enfermagem, inseridos em cenários assistenciais, sejam da iniciativa privada ou do Sistema Único de Saúde, convivem com diferentes cargas de trabalho e exposição a diferentes situações de risco ocupacional, carreando para si, o dúbio da produção da saúde e/ou do próprio desgaste físico (Gil, Camelo \& Laus, 2013). Neste caso, as ocorrências decorrentes do processo de trabalho no Centro de Material e Esterilização propiciam reações de instabilidade, exigindo, assim, novas adaptações e resiliência.

Atuando como Política Pública e processo educacional, a Educação Permanente em Saúde apresenta-se como uma proposta de ação estratégica capaz de contribuir para a transformação multiprofissional, a partir dos processos formativos, das práticas pedagógicas em saúde, visando à organização dos serviços, a partir do trabalho articulado entre o sistema de saúde em suas várias esferas de gestão, em que as instituições formadoras, apresentam envolvimento direto com a aprendizagem significativa no cenário do trabalho (Brasil, 2019; Peixoto et al. 2013).

Para interagir no mundo da vida e do trabalho, há de se inserir processos de reflexão crítica, que são a base para uma educação que considera o desenvolvimento da autonomia e da criatividade no ato de pensar, de sentir e de querer dos atores sociais (Brasil, 2019, Peixoto et al. 2013).

A Educação Permanente em Saúde ao atuar na transformação da práxis profissional, permite que o aprender e o ensinar, incorporem-se ao cotidiano coletivo e individual do trabalho.

Frente ao exposto, apresenta-se a questão norteadora: Com que ações a Educação Permanente em Saúde pode contribuir com vistas a aumentar a autoestima do trabalhador de enfermagem que atua no Centro de Material e Esterilização a partir da relevância do material processado, para a segurança do usuário e da equipe multidisciplinar de saúde? Nesse sentido, o objetivo desta pesquisa foi apontar a Educação Permanente em Saúde como instrumento de sensibilização com vistas a aumentar a autoestima do trabalhador de enfermagem do Centro de Material e Esterilização para gestores, coordenação de enfermagem e demais profissionais sobre os benefícios institucionais e sociais de um processo educacional ativo.

\section{Metodologia}

Trata-se de um recorte de um estudo exploratório com abordagem qualitativa, utilizando a técnica de análise de conteúdo (Câmara, 2013).

O estudo em questão, originado de dissertação, foi realizado em um Hospital Universitário, situado na região metropolitana II do Rio de Janeiro, Brasil, trazendo como cenário o Centro de Material e Esterilização. Os participantes foram 31 componentes da equipe de enfermagem: três Enfermeiros, 16 Técnicos e 12 Auxiliares de enfermagem, cumprindo escalas diurnas e noturnas de 12x60 horas ou escalas de seis horas diárias, exercendo respectivamente, atividades de coordenação e operacionalização de material e equipamentos, que contribuíram com a elaboração da pesquisa.

Foram definidos como critérios de inclusão dos participantes fazer parte do quadro efetivo da instituição. Excluíram-se os profissionais que se encontravam em licença médica, ou usufruindo de férias no período da coleta de dados.

Inicialmente, o convite para participar da pesquisa foi verbal, também foi informado aos participantes que a entrevista seria realizada no ambiente de trabalho, em local restrito. Os concordantes receberam informações quanto aos propósitos do estudo e, após assinatura do Termo de Consentimento Livre e Esclarecido. Como instrumento de coleta de dados, foi utilizado um questionário composto por questões abertas e fechadas, versando sobre a caracterização sociocultural dos participantes. Visando assegurar o sigilo e preservar a identidade dos participantes, os questionários receberam a vogal "E" associada à numeração arábica entre 01 a 31. 
Para a coleta de informações empíricas, que ocorreu entre setembro a outubro de 2014, utilizou-se como instrumento, uma entrevista composta por roteiro semiestruturado, gravada em aparelho eletrônico individualmente e posteriormente transcrita logo após a realização, possibilitando a pré-análise do material. O tempo de duração das entrevistas foi, em média, de 25 minutos.

A análise de conteúdo se deu a partir da transcrição dos dados obtidos via entrevistas e questionários respondidos, respeitando-se os três polos cronológicos: pré-análise; exploração do material; e tratamento dos resultados. As perguntas disparadoras foram: como é estar e a relação de você existir no Centro de Material e Esterilização? Quais os principais problemas vivenciados por você no Centro de Material e Esterilização que necessitam de ações educacionais? Gostaria de falar mais alguma coisa? Desvelando as seguintes categorias: Desagregação: comportamento resiliente e busca pelo protagonismo; Invisibilidade: quando o resultado sobrepõe à condição física do trabalhador e Enfrentamento: conflitos gerados pela baixa autoestima laboral.

Foram respeitados os aspectos éticos e legais, em conformidade com as determinações das Diretrizes de Normas Regulamentadoras de Pesquisas Envolvendo Seres Humanos, como o preconizado pela Resolução nº 466/2012 do Conselho Nacional de Saúde.

O protocolo de pesquisa foi submetido à apreciação do Comitê de Ética e Pesquisa da instituição, recebendo o CAEE: de $n^{\circ} 33739114.9 .0000 .5243$, parecer $n^{\circ} 777.581$.

\section{Resultados}

Dentre os 31 participantes, 24 pertencem ao sexo feminino e 7 ao masculino. Com relação à idade, 8 se encontram na faixa etária entre 41 e 50 anos, 13 entre 51 e 60 anos e 10 acima de 60 anos. Quanto ao tempo de atuação laboral, 9 compreendem o período entre 10 e 20 anos, 11 entre 21 e 30 anos, 09 entre 31 e 40 anos e 02 referem atuar na instituição, acima de 40 anos.

No que concerne ao tempo de exercício profissional no Centro de Material e Esterilização, 13 atuam de 1 a 4 anos, 12 de 05 a 08 anos, 02 de 09 a 13 anos e 04 acima de 13 anos.

Em relação ao nível de formação, a categoria profissional está assim distribuída: 03 enfermeiros; 16 técnicos de enfermagem e 12 são auxiliares de enfermagem. Ainda no quesito educação, no grupo de profissionais de nível superior, médio e fundamental, 09 referem ter pós-graduação, 04 graduação, 16 o nível médio e 02 o ensino fundamental.

Desta forma, do processo de análise dos relatos concernentes à problemática central do estudo emergiram as três categorias que irão subsidiar as discussões como descrito no método de estudo.

\section{Desagregação: comportamento resiliente e busca pelo protagonismo}

Objetivando ressaltar a importância e o papel de destaque do Centro de Material e Esterilização no âmbito de um serviço de saúde, buscou-se conhecer o sentimento dos profissionais da equipe de enfermagem, a partir da seguinte indagação: como é estar e a relação de você existir no Centro de Material e Esterilização?

[...] aqui é eu mando você obedece, eu falo e você faz. As ideias aqui, não são acatadas, não são absorvidas. (E10)

[...] precisava de ações que valorizassem mais enquanto servidor. Todos nós estamos desestimulados por tudo. (E5)

[...] deveria se olhar um pouquinho mais a parte emocional do grupo que trabalha aqui. (E8)

[...] as ideias, elas são ouvidas, mas infelizmente quando é para serem colocadas em prática, às soluções que são apresentadas, não são levadas em conta, deixando um pouco de frustração. (E9) 
[...] eu gostaria de falar, mas não vai adiantar, não vai melhorar, a nossa voz não tem muito peso, não se tem valorização pelo nosso trabalho, não temos suporte. (E13)

[...] a minha relação aqui é boa, mas gostava do meu setor anterior. (E30)

São notórios os sentimentos geradores de desconforto e desagregação embutidos nas impressões dos profissionais pesquisados, por não angariarem créditos em suas sugestões sobre o desenvolvimento do trabalho.

\section{Invisibilidade: quando o resultado sobrepõe à condição física do trabalhador}

Por vezes, o desenvolvimento do trabalho pode exercer influências sobre o corpo físico. Nesse contexto, buscou-se saber a impressão dos participantes, a partir da pergunta: quais os principais problemas vivenciados por você no Centro de Material e Esterilização que necessitam de ações educacionais?

[...] me sinto mal, sinto como se fosse um robô ou um burrinho de carga. A pessoa solicita, manda e você tem que obedecer. Quando não, ouve dizer: você está ganhando para isso. (E1)

[...] estou aqui por uma limitação de desempenho. Depois de 27anos de serviço eu vim conhecer o trabalho. Até então eu não conhecia. (E9)

[...] a realidade aqui é outra, completamente diferente.

Trabalhamos com equipamentos caríssimos e muito diversificados, material que você tem que estar atento. (E10)

[...] devido a minha limitação a minha chefia achou que seria um local bom para eu trabalhar (E26)

[...] aqui o serviço é pesado, deveriam colocar pessoas com mais saúde. Vêm doenças sérias para cá. (E21)

[...]. Porque o setor aqui é que desenvolve todo o hospital. Precisa de uma educação continuada para os auxiliares e técnicos e para os enfermeiros também. (E7)

\section{Enfrentamento: conflitos gerados pela baixa autoestima laboral}

Como anteriormente citado, o sentimento de inutilidade, gerado pela falta de valorização, em associação com a falta de diálogo, corroboram com que os profissionais, sintam-se descontentes, por não terem créditos em suas opiniões e conviverem com necessidades, muito aquém do esperado. Nesse contexto entabulou-se a pergunta: Gostaria de falar mais alguma coisa?

[...] o nosso trabalho poderia ser bem melhor, no momento ele está muito empobrecido, pouco valorizado e pouco respeitado $(E 1)$.

[...] o trabalho é muito cansativo. Atualmente trabalho na dobradura de roupas e fico muitas horas em pé (E2).

[...] o problema vivenciado pela nossa central é que nos consideramos um tanto quanto não representados junto aos demais setores do hospital (E9).

[...] para que haja melhoria é necessário que haja reflexão dos superiores, da diretoria, da gerência, para poder mudar. E não pensar que o centro de material e esterilização de hoje é o mesmo de 50 anos atrás. (E20) 


\section{Discussão}

Percebeu-se que o conceito atribuído ao setor ainda condiz com o pensamento ancestral de que nesse espaço - conforme o estudo denotou uma equipe composta predominantemente por mulheres idosas ou adentrando no processo de envelhecimento, já expressando barreiras de ordem, física, psíquica e emocional - vão poder aguardar na unidade o momento da tão sonhada aposentadoria.

Evidenciou-se o predomínio do gênero feminino, ligado a própria característica da profissão de enfermagem que veio a se feminilizar com o advento da modernidade. Atualmente, o cuidar diz respeito a homens e mulheres, apesar da divisão do trabalho, ainda remeter a uma construção social, de que as tarefas que abranjam sensibilidade, carinho, afetuosidade são consideradas femininas (Biasoli, 2016). Ainda sobre o predomínio do gênero feminino no mercado de trabalho, é um fenômeno que surgiu durante e após o pós-guerra, na Europa e Estados Unidos (Figueiredo, Mattar \& Abrão, 2013).

Frente à faixa etária, sabe-se que os profissionais com idade elevada, tendem a possuir maior experiência quanto ao desenvolvimento empírico do trabalho, o que torna relevante a atuação do enfermeiro na qualificação do trabalho no contexto Centro de Material e Esterilização diante de dificuldades apresentadas, garantindo assim, a eficácia dos processos, além de contribuir para a prevenção de infecções hospitalares (Ouriques \& Machado, 2013). Quanto mais o tempo passa, mais vivência o profissional levará para compor sua identidade no serviço, entretanto, esse fato pode alterar seu comportamento, pois o tempo de serviço tende a favorecer a manifestação de diferentes visões sobre uma prática, dissociada dos moldes científicos, e assim, produzir interferências nas relações interpessoais, na motivação ou no gosto pelo desempenho do trabalho, na disciplina hierarquizada (Morais et al. 2014).

Em relação ao nível de formação da equipe de enfermagem lotada no Centro de Material e Esterilização, a mesma é composta na maioria por profissionais de nível médio, porém percebeu-se uma dúbia condição, entre aqueles que relataram possuir graduação e pós-graduação, posto que, acredita-se que o ensino em nível de terceiro grau, possa contribuir com maiores expectativas e visão emancipatória, das atividades executadas em nível médio.

A qualificação profissional é o processo no qual o trabalhador adquire conhecimentos para o desempenho de determinada função visando melhor aproveitamento no exercício do trabalho. Assim, essa atitude de enfrentamento que inicialmente converge com o comportamento resiliente, descrito como processos de "superação" do indivíduo, perante crises e adversidades, não advém de ordem aleatória, porque como fruto dos recursos educacionais aplicados à qualificação profissional, são estimulados o interesse em protagonizar melhorias no trabalho (Fernandes, Curvo, \& Albuquerque, 2019; Athanázio et al. 2019; Oliveira et al. 2016). A esse respeito, a proposta da Educação Permanente em Saúde prevê que a mudança das estratégias de organização e do exercício profissional é constituída a partir da prática das equipes.

Como fonte de satisfação das necessidades humanas, o trabalho apresenta estreita relação com a autorrealização, com a preservação da saúde mental e qualidade dos relacionamentos interpessoais, sendo a ruptura de algum desses fatores, apontada como responsável pelo adoecimento biopsicossocial e causa de absenteísmo (Zorzanelli, Vieira \& Russo, 2016).

Quando se observa os resultados encontrados no item "Invisibilidade", quando o resultado se contrapõe à condição física do trabalhador", destaca-se que os serviços de saúde vêm ao longo dos anos vivenciando os efeitos da falta de concursos para a reposição de vagas, envelhecimento dos profissionais, baixos salários, situações que contrastam enormemente com a busca da produtividade adquirida a qualquer custo (Bernardes, Rocha \& Barboza, 2013; Rodrigues et al. 2013\& Bleck,2018). Conflitando assim, com os limites do ser humano, trazendo como resultado a despersonalização do indivíduo e o aumento do sofrimento psíquico no trabalho (Zorzanelli, Vieira \& Russo, 2016), em especial, para aqueles de faixa etária mais avançada e de baixa escolaridade. 
Embora mudanças substanciais e significativas tenham sido implementadas no mundo do trabalho tendo como base a tecnologia é pela resolutividade da mesma que, atualmente, pode-se entender que a falta de motivação, o desamparo, a desesperança, a passividade, a alienação, a depressão, a fadiga e o estresse são situações decorrentes do trabalho, comumente responsabilizadas por impor doença aos profissionais (Ceballos-Vásquez et al. 2015).

Ao predispor-se a ouvir, questionar e buscar transformar as decisões profissionais, acatando o paradigma inclusivo, a enfermeira estará atuando empaticamente com sua equipe, a partir dos dogmas estabelecidos pela Política Nacional de Educação Permanente em Saúde, quando infere ser por meio da problematização que os predisponentes humanos, responsáveis diretos pela qualidade do serviço prestado - em cada serviço de saúde, podem ser identificados e as necessidades de qualificação profissional das equipes garantidas (Oliveira et al. 2016).

A enfermeira coordenadora de serviços de um Centro de Material e Esterilização precisa se preparar, pois carreia uma grande responsabilidade sobre a dinâmica e exigências de um trabalho, em que devem ser consideradas as condições estruturais, as condições de entendimento (cognição) e aptidões dos profissionais sob seu comando para o desenvolvimento de atividades que não se encerram em si.

Já os resultados encontrados que se referem aos "Conflitos gerados pela baixa autoestima laboral” demonstram que, para amenizar o desgaste induzido por um ambiente de sobrecarga física, química, biológica e emocional (Costa et al. 2015), como o Centro de Material e Esterilização, acredita-se que a Educação Permanente em Saúde contribua ao colocar em evidência a formação e o desenvolvimento para o Sistema Único de Saúde, através da construção de estratégias que visem à educação no ambiente de trabalho - em meio ao cenário em que essas ações são desenvolvidas.

A Educação Permanente em Saúde constitui-se no processo de permanente aquisição de informações pelo trabalhador, de todo e qualquer conhecimento, por meio de escolarização formal ou não formal, de vivências, de experiências laborais e emocionais, no âmbito institucional ou fora dele. Com práticas pedagógicas baseadas em múltiplos fatores que compreendem os valores, as relações de poder, organização do trabalho, a aprendizagem significativa de adultos requer estratégias que enfoquem elementos que façam sentido para os envolvidos (Merhy, 2015).

Em decorrência desse estudo, sugere-se a Educação Permanente em Saúde, pela característica de valorizar os saberes trazidos por cada indivíduo, pelo recurso da problematização, dinâmicas de grupo e rodas de conversa, a construção e/ou reciclagem do conhecimento profissional. Assim, torna-se importante recurso na atualização e recriação de técnicas profissionais e, tende a contribuir no estímulo ao comportamento resiliente. Se condições de trabalho são dificilmente modificáveis no curto prazo, ao menos é possível intervir em condicionantes conjunturais. Neste sentido, a educação permanente em saúde permite que os indivíduos sejam empoderados e se tornem sujeitos de seu aprendizado e trabalho.

Pois, é a partir de uma análise rigorosa, sobre a condição biopsicointelectual, do trabalhador, é que se pode perceber a necessidade de segurança e de prevenção de agravos à saúde do mesmo, cujo produto final, é a salubridade de equipamentos e de materiais confiáveis, com os quais se espera contribuir no impedimento ou minimização de complicações à saúde e segurança de pacientes e de profissionais envolvidos na assistência direta.

\section{Conclusão}

A demanda do trabalho executado no Centro de Material e Esterilização, tendo por base a tecnologia e o volume de procedimentos realizados em saúde, apresenta-se em linha ascendente, enquanto que a contrapartida profissional vem em sentido contrário, permeando o pensamento de que a Educação Permanente em Saúde, devido ao seu enfoque inter e multiprofissional, surge como instrumento capaz de produzir transformações sobre as práticas e nos contextos de trabalho, fortalecendo a reflexão na ação, o trabalho em equipe e a capacidade de gestão sobre os próprios processos. 
Acredita-se que a falência do comportamento resiliente pode ser a alienação ou os transtornos de ordem biopsicossocial, aos quais requisita-se outras abordagens, pois as implicações para as boas práticas executadas na assistência direta pela enfermagem e equipe multidisciplinar de saúde contam em muito com a confiabilidade do material processado pela equipe de enfermagem do Centro de Material e Esterilização.

No que diz respeito às limitações do estudo, ocorre impossibilidades de generalização para outros contextos de trabalho, pois os resultados acima descritos não correspondem à totalidade e a realidade do que ocorre em todos os serviços de saúde do Rio de Janeiro. Porém, a exemplo de serviços de saúde na rede privada, sabe-se que não há o predomínio da faixa etária elevada no Centro de Material e Esterilização. Sugerimos a realização de novas pesquisas e discussões sobre a temática que envolvam o trabalho de enfermagem no Centro de Material e Esterilização, procurando dar visibilidade à unidade e aos profissionais que lá atuam.

\section{Agradecimentos}

Aos profissionais de enfermagem do Centro de Material e Esterilização que contribuíram para a realização da pesquisa.

Este estudo foi extraído da dissertação intitulada "Educação Permanente a trabalhadores do Centro de material e Esterilização: uma contribuição da enfermagem", apresentada em 2015 ao Curso de Mestrado Profissional em Ensino na Saúde da Escola de Enfermagem Aurora de Afonso Costa, Universidade Federal Fluminense, Rio de Janeiro/Brasil.

\section{Referências}

Associação Brasileira de Enfermeiros de Centro Cirúrgico, Recuperação Anestésica e Centro de Material e Esterilização (SOBECC). (2017). Diretrizes de práticas em enfermagem cirúrgica e processamento de produtos para a saúde. Barueri: Manole. São Paulo.

Athanázio, A. R., Teixeira, E. R., Cordeiro, B. C., Souza, L. M. C., Pereira, E. R., \& Silva, R. M. C. R. A. (2019). Aquisição de novos saberes pela equipe de enfermagem do centro de material e esterilização. In book: Discursos, Saberes e Práticas da Enfermagem; 107-15. Ed Atena. DOI: 10.22533/at.ed.75519261212.

Athanázio, A. R. Educação Permanente a Trabalhadores do Centro de Material e Esterilização: uma contribuição da enfermagem. (2015). Dissertação (Mestrado Profissional em Ensino na Saúde). Universidade Federal Fluminense, Rio de Janeiro, Brasil.

Bernardes, L. S., Rocha, I. C., \& Barboza, M. C. N. (2013). A insatisfação profissional dos enfermeiros de um hospital público no Centro Oeste. J Nurs Health, 3(1), 62-73.

Biasoli, P. K. (2016). Mulheres em cargos de gestão: dificuldades vinculadas ao gênero. Indic Econ FEE, 43(3), 125-40.

Bleck, N. (2018). Resilience During Difficult Times in Health Car. AACN Adv Crit Care, 29 (2), 115-17. https://doi.org/10.4037/aacnacc2018737.

Câmara, R. H. (2013). Análise de conteúdo: da teoria à prática em pesquisas sociais aplicadas às organizações. Rev Interinstitucional Psicol, 6(2), 179-91.

Ceballos-Vásquez, P., Rolo-González, G., Hérnandez-Fernaud, E., Díaz-Cabrera, D., Paravic-Klijn, T., \& Burgos-Moreno, M. (2015). Psychosocial factors and mental work load: a reality perceived by nurses in intensive care units. Rev Latino-Am Enfermagem, 23(2), 315-22. dx.doi.org/10.1590/01041169.0044.2557.

Cecilio, S. G., Brasil, L. G. B., Vilaça, C. P., Silva, S. M. F., Vargas, E. C., \& Torres, H. C. (2016). Psychosocial aspects of living with diabetes mellitus in promoting self-care. Rev Rene, 17(1), 44-51. http://dx.doi.org/10.15253/2175-6783.2016000100007.

Costa, C. C. P., Souza, N. V. D. O., Silva, P. A. S., Oliveira, E. B., \& Vieira, M. L. C. (2015). O trabalho na central de material: repercussões para a saúde dos trabalhadores de enfermagem. Rev Enfer. UERJ, 23(4), 533-39. http://dx.doi.org/10.12957/reuerj.2015.15934.

Cruz, E. J. E. R., Souza, N. V. D. O., Amorim, L. K. A., Pires, A. S., Gonçalves, F. G. A., \& Cunha, L. P. (2018). Resiliência como objeto de estudo da saúde do trabalhador: uma revisão narrativa. Rev Fundam Care Onlin, 10(1), 283-8. https://doi.org/10.9789/2175-5361.2018.

Deuster, P., \& Silverman, M.N. (2013). Physical fitness: a pathway to health and resilience. US Med Dep J, $24-35$.

Fernandes, T., Curvo, A. D., \& Albuquerque, R. A. F. (2019). Resilience and socialization between public servers: a case study at the Federal University of Mato Grosso - UFMT. Research, Society and Development, 8(4), e4784902. https://doi.org/10.33448/rsd-v8i4.902.

Figueiredo, S. F., Mattar, M. J. G., \& Abrão, A. C. F. V. (2013). Baby-Friendly Hospital: prevalence of exclusive breastfeeding at 6 months and intervening factors. Rev Esc Enferm USP, 47(6), 1291-7. dx.doi.org/10.1590/S0080-623420130000600006. 
Research, Society and Development, v. 10, n. 3, e31910313386, 2021

(CC BY 4.0) | ISSN 2525-3409 | DOI: http://dx.doi.org/10.33448/rsd-v10i3.13386

Fusco, S. F. B., \& Spiri, W. C. (2014). Analysis of quality indicators of central sterile supply departments at accredited public hospitals. Texto Contexto Enferm, 23(2), 426-33. dx.doi.org/10.1590/0104-07072014001570013.

Gil, R. F., Camelo, H. S., \& Laus, A. M. (2013). Nursing tasks in the Material Storage Center of hospital institutions. Texto Contexto Enferm, 22 (4), $927-34$. doi: http://dx.doi.org/10.1590/S0104-07072013000400008

Merhy, E. E. (2015). Educação permanente em movimento - uma política de reconhecimento e cooperação, ativando os encontros do cotidiano no mundo do trabalho em saúde, questões para os gestores, trabalhadores e quem mais quiser se ver nisso. Saúde Redes, 1(1), 7-14. http://revista. redeunida.org.br/ojs/index.php/rede-unida/ article/view/309/15.

Ministério da Saúde, Brasil. (2012). Agência Nacional de Vigilância Sanitária (ANVISA). Resolução - RDC no 15, de 15 de março de 2012. Diário Oficial da União (DOU) n n 54 , de 19 de março de 2012. 43-6.

Ministério da Saúde, Brasil. (2019). Política Nacional de Educação Permanente em Saúde. Brasília: Ministério da Saúde.

Morais, M. P., Martins, J. T., Robazzi, M. L. C. C., \& Cardelli, A. A. M. (2014). Dissatisfaction in the work of nurses of a university hospital. Cogitare Enferm, 19(2), 316-22. https://revistas.ufpr.br/cogitare/article/view/31529/22759

Oliveira, M. P. R., Menezes, I. H. C. F., Sousa, L. M., \& Peixoto, M. R. G. (2016). Training and Qualification of Health Professionals: Factors associated to the Quality of Primary Care. Rev Bras Edu Méd, 40(4), 547-59. dx.doi.org/10.1590/1981-52712015v40n4e02492014.

Ouriques, M. C., \& Machado, M. E. (2013). Nursing in the process of sterilization of materials. Texto Contexto Enferm, 22(3), 695-03. http://dx.doi.org/10.1590/S0104-07072013000300016.

Oviedo, R. A., \& Czeresnia, D. (2015). The concept of vulnerability and its biosocial nature. Interface, 19(53), 237-50. https://doi.org/10.1590/180757622014.0436 .

Peixoto, L. S., Gonçalves, L. C., Costa, T. D., Melo, C. M. T, Cavalcanti, A. C. D., \& Cortez, E. A. (2013). Educación permanente, continuada y de servicio: desvelando sus conceptos. Enferm Glob, 12(1), 307-22. http://revistas.um.es/eglobal/article/view/141801

Portella, A., \& Liberalesso, N. A. (2015). Resilience in aging: literature review. Ciênc Saúde Coletiva, 20 (5), 1475-95. dx.doi.org/10.1590/141381232015205.00502014.

Rodrigues, D. P., Athanázio, A. R., Cortez, E. A., Teixeira, E.R., \& Alves, V. H. (2013). Estresse na Unidade de Terapia Intensiva: revisão integrativa. Rev enferm UFPE on line, 7(esp), 1595-04. 10.5205/reuol.4134-32743-1-SM-1.0705esp201315.

Silva, J. M. B., Loureiro, L. H., Silva, I. C. M., \& Novaes, M. L. (2020). Coronavirus and the disinfection and reprocessing protocols of hospital articles. Research, Society and Development, 9(9), e29996187. https://doi.org/10.33448/rsd-v9i9.6187.

Zorzanelli, R., Vieira, I., \& Russo, J. Á. (2016). Several names for tiredness: emergent categories and their relationship with the world of work. Interface Botucatu, 20(56), 77-88. dx.doi.org/10.1590/1807-57622015.0240. 